\title{
Left bundle branch area pacing lead implantation using an uninterrupted monitoring of endocardial signals.
}

\author{
Marek Jastrzebski ${ }^{1}$ \\ ${ }^{1}$ Uniwersytet Jagiellonski w Krakowie I Klinika Kardiologii i Nadcisnienia Tetniczego
}

February 2, 2022

\begin{abstract}
An 82-year old woman with third degree atrioventricular block underwent left bundle branch area pacing (LBBAP) lead implantation

\section{Hosted file}

LBBAP implantation guided by COI + figuers.doc available at https://authorea.com/users/ 422495/articles/555100-left-bundle-branch-area-pacing-lead-implantation-using-anuninterrupted-monitoring-of-endocardial-signals
\end{abstract}

